\title{
Performance Analysis of RoFSO Links with Diversity Reception for Transmission of OFDM Signals Under Correlated Log-normal Fading Channels
}

\author{
Fan Bai, Yuwei Su and Takuro Sato
}

Graduate School of Globe Information and Telecommunication Studies, Waseda University, Tokyo 169-0072, Japan

E-mail:baifan@ruri.waseda.jp,bruce.suyuwei@yahoo.co.jp,t-sato@waseda.jp

Received: October 14, 2014;

Accepted: November 10, 2014

\begin{abstract}
Free space optical (FSO) communication has been receiving growing attention with recent commercialization successes as a cost-effective and high bandwidth optical access technique. Meanwhile, FSO communication has been regard as an attractive solution to bridging the gap between the wireless communications and optical fiber communications. However, a significant performance degradation in FSO communication system due to the atmospheric turbulence impairs the transmission performance improvement. FSO system employing the spatial diversity technique can be used to mitigate the effect of turbulence and improve the transmission performance. In this paper, a novel analytical approach is presented to evaluate the transmission performance of OFDM-FSO system with diversity reception considering effect of channel correlation. A detailed mathematical model for OFDM-FSO system over turbulent channel modeled by correlated Log-normal distribution is provided. Then, We derive the signal-to-noise ratio (SNR), bit error ratio (BER) and outage probability expressions taking into account the diversity combining schemes (i.e. MRC, EGC), effects of atmospheric turbulence, channel correlation and aperture size of receiver lens. The results of this
\end{abstract}

Journal of ICT, Vol. 2, 129-150.

doi: $10.13052 /$ jicts2245-800X.224

(c) 2014 River Publishers. All rights reserved. 
study show that the most significant parameters that degrade the system performance. Furthermore, the obtained numerical results can be useful for designing, evaluating and enhancing the FSO system's ability to transmit wireless signal under actual conditions.

Keywords: Free Space Optical (FSO), Orthogonal Frequency Division Multiplexing (OFDM), spatial diversity, channel correlation, atmospheric turbulence, Maximum Ratio Combining (MRC), Equal Gain Combining (EGC).

\section{Introduction}

Recently, with the growing demand on the high-speed and high-quality applications for the communication devices such as high-definition television (HDTV), video programs and so on, we observe an explosion in the network traffic. Free space optical (FSO) system known as optical wireless communication (OWC) application was proposed for its potential high data rate capacity, low cost, and wide bandwidth on unregulated spectra. At the same time, transmission of radio frequency (RF) signals by means of optical fiber links, which are commonly referred to as radio over fiber (RoF), has been utilized for many years as a cost effective and high-capacity solution to connect the wireless signal and optical fiber communication [1]. FSO link can conveniently be used to transmit RF signals, which are similar to RoF but excluding the fiber medium as radio-on free space optics (RoFSO) [3]. Thus, the commercial FSO systems can be seem as a good candidate for supporting the heterogeneous services in the next generation access networks. In the mean time, ITU-T also published the first ITU-T recommendation in the area of G.640 for FSO application in the practical issue [2].

For the case of FSO link model, one of the main problems that affect the transmission performance is irradiance fluctuations induced by the atmospheric turbulence. This irradiance known as the optical scintillation, can cause the power losses [5]. It is the major performance impairment in FSO system. Therefore, it is importance to apply efficient techniques to mitigate the channel fading caused by atmospheric turbulence.

Spatial diversity is an efficient solution to mitigate channel fading by using the multiple apertures at the receiver (SIMO) or transmitter side (MISO) or combination (MIMO) [9]. Further, for the 5G communication technology, massive MIMO is regard as one of the important solution. However, diversity techniques are most efficient under the conditions of uncorrelated fading on the 
sub-channels $[6,10]$. In the previous studies, any two adjacent antennas must be sufficiently far apart as independent case due to channel correlation will impair the transmission performance improvement [7]. Therefore, one of the key points is determining the relation between sub-channel correlation among each adjacent antenna over the long transmission distance and transmission performance of FSO system.

Orthogonal frequency division multiplexing (OFDM) is a widely applied technique for wireless communications that divides the spectrum into a number of equally spaced sub-channels and carries a portion of a users information on each channel [5]. OFDM/OFDMA, which is the air interface for the Long Term Evolution (LTE) in wireless communication systems, is regard as one of important broadband application areas using RoF technology. Meanwhile, OFDM has been adopted in several high-speed digital communication standards such as digital terrestrial TV broadcasting and the IEEE 802.11 local area network (LAN) and IEEE 802.16 standards [8].

Furthermore, in order to get an insight into the atmospheric channel statistical models effects to the system performance, we consider the correlated Log-normal distribution due to its excellent match to multiple sub-channel links over weak turbulence fading channel. Since the FSO link can be modeled as diversity reception, an efficient diversity combining scheme is required to improve the system performance. As we know, maximum ratio combining (MRC) can provide an optimum combining performance, but implementation complexities are inherent, and system is extremely sensitive to channel estimation error. Equal gain combining (EGC) is inefficient for system with branches having acutely low SNR conditions $[8,9]$

In this paper, we propose a OFDM-FSO system with dual diversity reception over correlated Log-normal distribution fading channel. The goal of this paper is to analyze and explore the potential of OFDM-FSO system with diversity reception for use in high-performance high-speed transmission. Then, we investigate channel correlation effects on transmission performance, in terms of averaging bit error ratio (BER), signal-to-noise ratio (SNR), and outage probability $(\mathrm{OP})$. We take into consideration the effect of diversity combining scheme, aperture size of receiver lens and number of diversity reception. In this analysis, the use of spatial reception configuration with OFDM modulation can be seem as a countermeasure for the mitigation of scintillation and enhancing the performance of FSO systems in operation environments. Besides, our theoretical study provides guidelines to optimally configure the actual system design by applying an optimum transmission performance. 
The rest of this paper is organized as follows. In Section 2, we derive the expressions for the channel correlation coefficient for a dual diversity reception based on the plane wave model. Then, we model the probability density function of bivariate Log-normal random variables based on diversity reception in Section 3. In the Section 4, we introduce the dual diversity OFDM-FSO system structure and present the mathematical modeling for the transmission of OFDM signal over fading channel modeled by correlated Log-normal distribution. In the Section 5, we provide the numerical results to analyze and discuss the effect of the spatial diversity on the OFDM-FSO link over turbulent channel. Finally, we give the conclusion in Section 6.

\section{Channel Correlation in Atmospheric FSO Links with Diversity Reception}

In this section, we review the theories used to derive the expression for the channel correlation for diversity reception over turbulent channel. Then, we present the mathematical modeling for the joint probability density function (PDF) which represents two Log-normal random variables (RVs) of the light intensity by turbulence channel.

The most serious effects that severely affect the propagation of a laser beam propagating through the atmosphere are caused by variations of the refractive index in turbulent channel [4]. An important parameter that describes the strength of turbulence is the scintillation index: $\sigma_{I}^{2}=$ $\left\langle I^{2}\right\rangle /\langle I\rangle^{2}-1$, with $I$ being the intensity of optical wave. In fact, atmospheric turbulence as an important influence factor which it can cause the intensity fluctuation in the received signal level and leads to sharp increase in the bit error in a FSO communication link. Intensity fluctuation at a received power variance that depends on the size of the receive aperture, most of previous literatures proposed reduce the power variance by increasing the diameter of aperture as aperture averaging (AA) method [5, 6]. Aperture averaging can be seen as a simple form of spatial diversity when the receiver lens aperture is larger than the fading correlation length [8].

In this paper, we used spatial domain technique model a simple dual diversity reception FSO system and collect the OFDM signal at different two positions. Consider a dual diversity reception FSO link transmitted by plane wave model as show in Figure 1. We define the channel correlation coefficient $\rho_{12}$ between any component receivers, denoted 1 th receiver and 2 th receiver: 


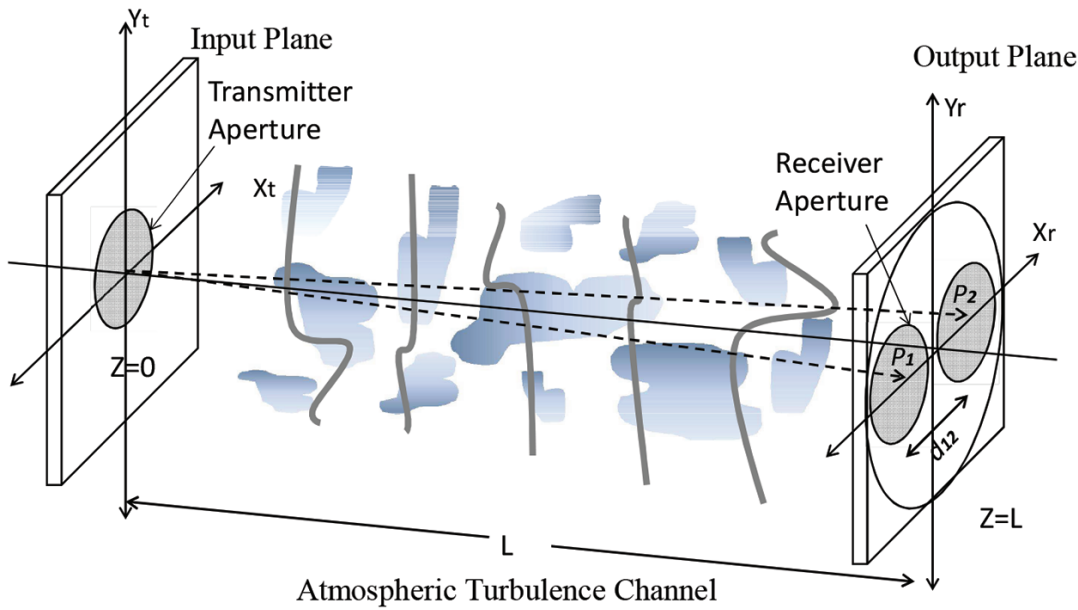

Figure 1 A dual diversity reception based on plane wave model

$$
\rho_{12}\left(d_{12}, D\right)=\frac{B_{I, 12}\left(I_{1}, I_{2}, d_{12}, D\right)}{\sqrt{\sigma_{1}^{2} \sigma_{2}^{2}}}
$$

with $d_{12}$ being the separation distance of two points in the adjacent receiver aperture plane, $D$ is aperture diameter. In this paper, $d_{12}$ is a center-to-center separation distance. $B_{I, 12}$ and $\sigma_{j}^{2}(j=1,2)$ are spatial covariance function of the irradiance and scintillation index of the $j$ th diversity receiver. In order to characterize the irradiance and the scintillation index of receiver induced channel fading, we use the light irradiance spatial co variance function [5]:

$$
\begin{gathered}
B_{I, 12}\left(I_{1}, I_{2}, d_{12}, D\right)=\frac{\left\langle I_{1} I_{2}\right\rangle}{\left\langle I_{1}\right\rangle\left\langle I_{2}\right\rangle}-1 \\
B_{I, i, j}\left(I_{i}, I_{j}, 0, D\right)=\sigma_{i=j}^{2}(D)=\frac{\left\langle I_{j}^{2}\right\rangle}{\left\langle I_{j}\right\rangle^{2}}-1
\end{gathered}
$$

where $\left\langle\right.$. $>$ for ensemble average over different turbulence disorder and $I_{j}$ is the light intensity collected by the $j$ th receiver. Previous equations indicate that the irradiance spatial covariance function is a more general statistic model 
that includes the scintillation index as a special case when the space distance between adjacent point as zero $\left(d_{12}=0\right)$.

Furthermore, based on the extended Rytov theory, we can also use the logamplitude $X$ and log-amplitude variance $\sigma_{X}^{2}$ of the optical wave represents two points on-axis log-amplitude fluctuation covariance function, the logamplitude variance is defined by [5]

$$
\begin{aligned}
B_{X, 12}\left(r_{1}, r_{2}, L\right) & =\left\langle\left(X_{1} X_{2}\right)^{2}\right\rangle-\left\langle\left(X_{1} X_{2}\right)\right\rangle^{2} \\
& =\frac{1}{2} \operatorname{Re}\left[E_{2}\left(r_{1}, r_{2}, L\right)+E_{3}\left(r_{1}, r_{2}, L\right)\right]
\end{aligned}
$$

when the equation indicates the on the optical axis point log-amplitude fluctuation covariance means that the valued of the transverse vector at output plane with $r_{j}=0$. Where $\operatorname{Re}\left(\right.$.) denotes the real part of $X$, and $E_{2}, E_{3}$ are two second-order statistical moments given by $[5,13]$

$$
\begin{gathered}
E_{2}(0,0, L)=4 \pi^{2} k^{2} L \int_{0}^{1} \int_{0}^{\infty} k \Phi_{n . e f f}(k) \\
\exp \left(-\frac{k^{2} D^{2}}{16}\right) J_{0}\left(K d_{12}\right) d k d \xi \\
E_{3}(0,0, L)=-4 \pi^{2} k^{2} L \int_{0}^{1} \int_{0}^{\infty} k \Phi_{n . e f f}(k) \\
\exp \left(-\frac{k^{2} D^{2}}{16}-i \frac{L \xi}{k}\right) J_{0}\left(K d_{12}\right) d k d \xi
\end{gathered}
$$

where $\xi=1-z / L$ and $J_{0}($.$) is Bessel function of the first kind and zero order,$ $k$ is optical wave number. The $L$ represent transmission distance. The effective atmospheric spectrum $\Phi_{n . e f f}(k)$ is used so that the covariance function is valid under all turbulence conditions. When the effective atmospheric spectrum $\Phi_{n . e f f}(k)$ under neglect inner-scale and outer-scale situation is defined as [4]

$$
\Phi_{n . e f f}(k)=0.033 C_{n}^{2} k^{-11 / 3}\left[\exp \left(-\frac{k^{2}}{k_{X .0}^{2}}\right)+\frac{k^{11 / 3}}{\left(k^{2}+k_{Y .0}^{2}\right)^{11 / 6}}\right]
$$

with

$$
k_{X .0}^{2}=\frac{k}{L} \frac{2.61}{1+1.1 \sigma_{R}^{2}}
$$




$$
k_{Y .0}^{2}=\frac{3 k}{L}\left(1+0.69 \sigma_{R}^{12 / 5}\right)
$$

The $k_{X .0}$ and $k_{Y .0}$ represent the low-pass and high-pass spatial frequency cutoffs, $\sigma_{R}^{2}$ for turbulence strength. When the log-amplitude variance is sufficiently small, and making $r_{1}=r_{2}=0$ for the on-axis values, the scintillation index is related by

$$
B_{I, j j}\left(I_{j}, I_{j}, 0, D\right)=\sigma_{j}^{2}(D)=\exp \left[4 B_{X, 12}\left(r_{1}, r_{2}, L\right)\right]-1
$$

Then, when the observation points on the optical axis, the equation (2) can be changed to

$$
\begin{aligned}
B_{I, 12}\left(I_{1}, I_{2}, d_{12}, D\right)= & \exp \left\{2 \operatorname{Re}\left[E_{2}(0,0, L)+E_{3}(0,0, L)\right]\right\}-1 \\
= & \exp \left\{8 \pi^{2} k^{2} L \int_{0}^{1} \int_{0}^{\infty} \Phi_{n . e f f}(k) \exp \left(-\frac{k^{2} D^{2}}{16}\right)\right. \\
& \left.\times J_{0}\left(k d_{12}\right)\left[1-\cos \left(\frac{L k^{2} \xi}{k}\right)\right] d k d \xi\right\}-1
\end{aligned}
$$

\section{Correlated Log-normal Distribution Model}

Theory reliably of FSO system operating in presence of atmospheric turbulence can be based on a mathematical model of the probability density function of randomly light intensity irradiance that describes the system's behavior across the different turbulence strength regimes [6]. In this paper, we model the correlated Log-normal distribution represents combine faded of intensity irradiance, then, we investigate the effect of channel correlation to system performance under weak turbulence regime. Based on previous literatures [5, 13], which single receiver Log-normal probability density function (PDF) of light intensity fading is given by

$$
f_{I}(I)=\frac{1}{2 I} \frac{1}{\left(2 \pi \sigma_{X}^{2}\right)^{1 / 2}} \exp \left\{-\frac{\left(\ln (I)-\ln \left(I_{0}\right)\right)^{2}}{8 \sigma_{X}^{2}}\right\}
$$

where $I_{0}$ denotes the light intensity in the absence of turbulence, $I=I_{0} \exp (2 X-2\langle X\rangle)$. Hence, a diversity reception FSO system where optical signals collected by multiple apertures undergo diversity combining, we model the spatial covariance matrix $K$ as 


$$
K=\left[\begin{array}{cccc}
k_{11} & k_{12} & \cdots & k_{1 n} \\
k_{21} & k_{22} & \cdots & k_{2 n} \\
\vdots & \vdots & \ddots & \vdots \\
k_{n 1} & k_{n 2} & \cdots & k_{n n}
\end{array}\right]
$$

where $k_{i j}=B_{I, i j}$ is spatial covariance function, $B_{I, i j}=\sigma_{j}^{2}(D)$ when the $i=j$ as equation (2) and (3). This spatial covariance matrix can be represented multiple transceivers case to determine the effect of correlation among the sub-channels. Then, we assume correlated Log-normal distribution function of the light intensity of dual sub-channels is given by

$$
\begin{aligned}
f_{I_{1}, I_{2}}\left(I_{1}, I_{2}\right) & =\frac{1}{2^{2} I_{1} I_{2}} \frac{1}{2 \pi \sigma_{X 1} \sigma_{X 2} \sqrt{1-\rho_{12}^{2}}} \\
& \times \exp \left\{-\frac{1}{8\left(1-\rho_{12}^{2}\right)}\left[\frac{\left(\ln I_{1}-\ln I_{1,0}\right)^{2}}{\sigma_{X 1}^{2}}+\frac{\left(\ln I_{2}-\ln I_{2,0}\right)^{2}}{\sigma_{X 2}^{2}}\right.\right. \\
& \left.\left.-2 \rho_{12} \frac{\left(\ln I_{1}-\ln I_{1,0}\right)\left(\ln I_{2}-\ln I_{2,0}\right)}{\sigma_{X 1} \sigma_{X 2}}\right]\right\}
\end{aligned}
$$

where $I_{1,0}$ and $I_{2,0}$ are the received light intensity at the 1 th receiver and 2 th receiver in the absence of turbulence situation.

\section{Performance Analysis of a Atmospheric OFDM-FSO Link with Diversity Reception}

In this paper, the main focus is to establish an analytical model able to characterize the OFDM signal transmission through an intensity modulation/directdetection (IM/DD) FSO link. We derive the expressions of the average signal-to-noise ratio (SNR), average bit error ratio (BER) and outage probability, which analysis considers on the effect of scintillation, both of MRC and EGC combining schemes and aperture size of receiver lens in this section. Then, a dual diversity reception OFDM-FSO system is considered where the OFDM signal is transmitted via single aperture and collected by two apertures $(j=2)$ over turbulent channel modeled by correlated Log-normal distribution with additive white Gaussian noise (AWGN). Figure 2 shows OFDM-FSO diversity receivers system architecture. The OFDM signal for $N_{s}$ sub-carriers, after up-conversion can be written as 


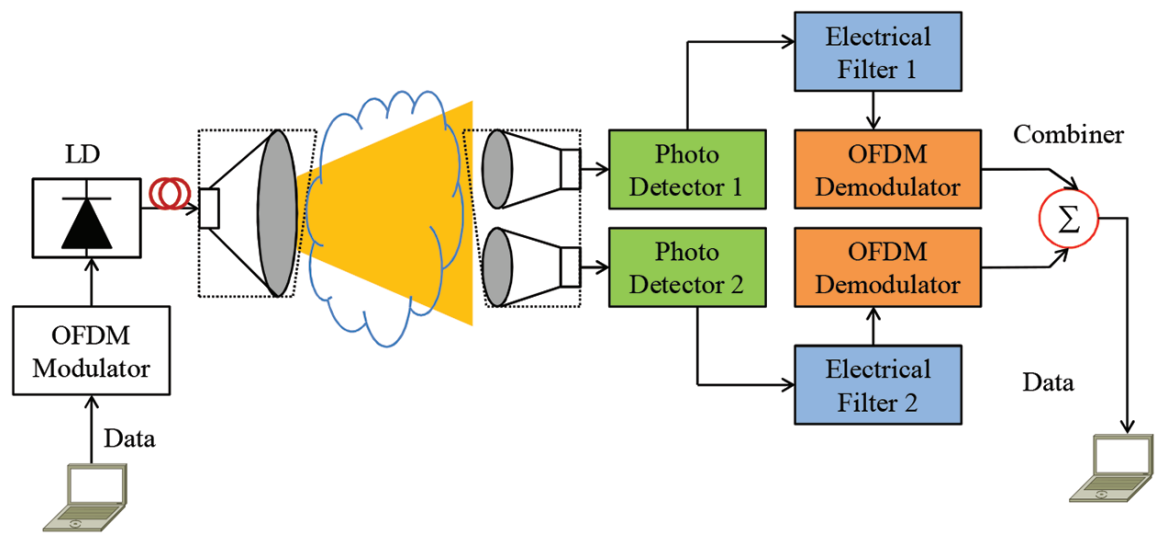

Figure 2 OFDM-FSO system with dual diversity reception over atmospheric turbulence channel

$$
S_{O F D M}(t)=\sum_{n-0}^{N_{s}-1} X_{n} \exp \left\{j\left(\omega_{n}+2 \pi f_{c}\right) t\right\}, 0 \leq t<T_{s}
$$

Where $\omega_{n}$ are the set of orthogonal sub-carriers frequency, $T_{S}$ is the OFDM symbol duration, $X_{n}=a_{n}+j b_{n}$ is complex data symbol in the nth subcarrier, with an and bn the in-phase and quadrature modulation symbols. The first raw data is mapped according to different types of modulation techniques. Each symbol $X_{n}$ is amplitude modulated on orthogonal sub-carriers. The signal $S_{O F D M}(\mathrm{t})$ is then used to modulate the optical intensity of laser-diode (LD) to be transmitted through the fiber optics. In this paper, we neglect the inter-modulation distortion (IMD). Then, the optical power output from the LD can be expressed as

$$
P(t)=P_{t}\left(1+\sum_{n=0}^{N-1} m_{n} S_{O F D M}(t)\right)
$$

where $P_{t}$ is average transmitted optical power, $m_{n}$ is optical modulation index on per sub-carrier. At the receiver side, the received optical power in the presence of turbulence at the photo detector input can be express as

$$
P_{r . F S O}(t)=P(t) L_{l o s s} X+n_{F S O}(t)
$$


where $L_{\text {loss }}$ is the sum of losses due to atmospheric turbulence, geometrical loss and pointing error. The $n_{F S O}(t)$ characterizes the additive white Gaussian noise (AWGN), $X$ quantifies the variation of the signal fading due to atmospheric turbulence effects.

Then, the received optical signal convert to the electrical signal by photodetector (PD), and the FSO noise can be filtered in the PD. Then, signal pass through the electrical filter and OFDM demodulator. Assuming the total output photo current of the combiner in the presence of turbulence can be expressed as follow

$$
i\left(t, I_{1}, I_{2}, X\right)=I_{p h}\left(I_{1}, I_{2}\right)\left(1+\sum_{n=0}^{N-1} m_{n} S_{O F D M, 1,2}(t)\right)+n_{\text {opt }}, 1,2(t)
$$

where $I_{p h}=R_{D} P_{t} L_{\text {loss }} X$ is dc of the received photo current depend on received light intensity, and including $I_{1}$ and $I_{2}$ received by each receiver, $R_{D}$ is detector responsivity, $m_{n}$ is optical modulation index on per subcarrier, and $n_{\text {opt }}(t)$ is the sum of thermal noise, shot noise and is modeled as Gaussian white random process with zero mean and variance $\sigma_{N}^{2}=N_{0} / 2$. The total noise power is given by

$$
N_{0}\left(I_{1}, I_{2}\right)=\frac{4 K_{B} T_{a b s} F_{e}}{R_{L}}+2 q I_{p h}\left(I_{1}, I_{2}\right)+(R I N) I_{p h}^{2}\left(I_{1}, I_{2}\right)
$$

where $K_{B}$ is the Boltzmanns constant, $T_{a b s}$ is the absolute temperature, $F_{e}$ is the noise figure of the receiver electronics, $R I N$ is relative intensity noise, $R_{L}$ is the PD load resistor, and $q$ is the electron charge.

In this analysis, we compare the performance of dual diversity reception with two diversity combining schemes, maximal ratio combining (MRC) and equal gain combining (EGC). In the presence of turbulence, the instantaneous electrical signal-noise-to ratio (SNR) at the output of the diversity combiner using MRC and EGC are given by [13]

$$
\begin{gathered}
\gamma_{\text {total.MRC }}\left(I_{1}, I_{2}, X_{1}^{2}, X_{2}^{2}\right)=\sum_{j=1}^{J} \gamma_{j}\left(X_{j}^{2}\right),(j=2) \\
\gamma_{\text {total.EGC }}\left(I_{1}, I_{2}, X_{1}^{2}, X_{2}^{2}\right)=\left(\sqrt{\gamma_{1}\left(X_{1}^{2}\right)}+\sqrt{\gamma_{2}\left(X_{2}^{2}\right)}\right)^{2} / 2
\end{gathered}
$$


where $\gamma_{j}$ is instantaneous SNR of $j$ th receiver in the turbulent channel. Thus, the received photo current is proportional to $X$ and has same statistic in the presence of the turbulence. Moreover, consider a dual diversity reception OFDM-FSO system using M-QAM modulation where the subchannel collected light intensity $\left(I_{1}, I_{2}\right)$ follow a correlated Log-normal distribution. Taking into consideration the correlation between the receivers with log-amplitude fluctuation and represented by $X$, thus, the equation (14) can be modified as follow

$$
\begin{aligned}
f_{X 1, X 2}(X 1, X 2)= & \frac{1}{2 \pi \sigma_{X 1} \sigma_{X 2} \sqrt{1-\rho_{12}^{2}}} \\
& \times \exp \left\{-\frac{1}{2\left(1-\rho_{12}^{2}\right)}\left[\frac{\left(X_{1}-\left\langle X_{1}\right\rangle\right)^{2}}{\sigma_{X 1}^{2}}+\frac{\left(X_{2}-\left\langle X_{2}\right\rangle\right)^{2}}{\sigma_{X 2}^{2}}\right.\right. \\
& \left.\left.-2 \rho_{12} \frac{\left(X_{1}-\left\langle X_{1}\right\rangle\right)\left(X_{2}-\left\langle X_{2}\right\rangle\right)}{\sigma_{X 1} \sigma_{X 2}}\right]\right\}
\end{aligned}
$$

Finally, the total average BER for the received QAM-OFDM signal, where $M=2^{n}$ and $n$ is an even number, is given by [13]

$$
\left\langle B E R_{\text {total }}\right\rangle=\int_{0}^{\infty} \int_{0}^{\infty} f_{X_{1}, X_{2}}\left(X_{1}, X_{2}\right) B E R_{\text {total }}\left(X_{1}^{2}, X_{2}^{2}\right) d X_{1} d X_{2}
$$

where instantaneous electrical $B E R_{\text {total }}$ is

$$
B E R_{\text {total }}\left(X_{1}^{2}, X_{2}^{2}\right)=\frac{2\left(1-\sqrt{M}^{-1}\right)}{\log _{2}^{M}} \operatorname{erfc}\left(\sqrt{\frac{3 \gamma_{\text {total }}\left(X_{1}^{2}, X_{2}^{2}\right)}{2(M-1)}}\right)
$$

The case of MRC reception combining scheme with dual diversity reception, the electrical SNR in the presence of turbulence can be written as [13]

$$
\gamma_{\text {total.MRC }}\left(X_{1}^{2}, X_{2}^{2}\right)=\frac{0.5 m_{n}^{2}\left(I_{p h .1}^{2}\left(I_{1}, X_{1}^{2}\right)+I_{p h .2}^{2}\left(I_{2}, X_{2}^{2}\right)\right)}{N_{0} B_{e}}
$$

and EGC reception combining scheme can be modeled by [13]

$$
\gamma_{\text {total.EGC }}\left(X_{1}^{2}, X_{2}^{2}\right)=\left[\frac{\sqrt{0.5} m_{n}\left(I_{p h .1}^{2}\left(I_{1}, X_{1}^{2}\right)+I_{p h .2}^{2}\left(I_{2}, X_{2}^{2}\right)\right)}{\sqrt{2 N_{0} B_{e}}}\right]^{2}
$$


where $I_{p h . j}$ is the function related to $I_{j}$ and $X_{j}$ for the jth receiver, at the same time, $I_{p h . j}$ also follow the same distribution model of $X_{j}$, and $B_{e}$ is electrical filter bandwidth.

The outage probability is a commonly used performance metric in fading channels. It is defined as the probability that instantaneous $\operatorname{SNR}\left(\gamma_{\text {total }}\right)$ falls below a specified threshold SNR $\left(\gamma_{t h}\right)$, which represents a value of the SNR above which the quality of the channel is satisfactory. It is a useful method to evaluate the effect of the channel fading cause to the atmospheric turbulence on the system performance. The outage probability for a given threshold SNR $\left(\gamma_{t h}\right)$ can be defined as

$$
P_{\text {out }}\left(\gamma_{t h}\right)=P\left[\gamma_{\text {total }}<\gamma_{t h}\right]
$$

For the case of MRC, the outage probability is given by [13]

$$
P_{\text {out }}\left(\gamma_{t h}\right)=\int_{0}^{\gamma_{t h}} \int_{0}^{\gamma_{t h}-\gamma_{2}} f\left(\gamma_{1}, \gamma_{2}\right) d \gamma_{1} d \gamma_{2}
$$

where $f\left(\gamma_{1}, \gamma_{2}\right)$ is the function of SNR follow the correlated Log-normal distribution.

\section{Numerical Results}

In this section, we present numerical results for the transmission performance of OFDM-FSO system with dual diversity reception under correlated Lognormal distribution. The main simulation parameters used in the numerical calculation are shown in Table 1.

Since the character of the channel correlation coefficient has been investigate in several works $[6,13]$, we prefer to recall the general points that will be useful while analyze the transmission performance to follow. Figure 3 shows typical curves for the channel correlation coefficient $\rho_{12}$ as a function of separation distance under variation turbulence strength regimes represented by $\sigma_{R}^{2}$, and aperture size is given a fixed value $D=2 \mathrm{~cm}$. In this analysis, the optical wavelength $\lambda=1550 \mathrm{~nm}$ and transmission distance is $2 \mathrm{~km}$. From the graph, we can clear see that the channel correlation $\rho_{12}$ decreases with increasing separation distance $d_{12}$, and the curves go cross a zero point where the channel correlation length $\rho_{c}$ is defined (i.e. $\rho_{12}=0$ ) under the variation turbulence strength $\sigma_{R}^{2}$ with fixed aperture diameter $D$. In general, the channel 
Table 1 Numerical Parameters

\begin{tabular}{ll} 
Operating wavelength $\lambda$ & $1550 \mathrm{~nm}$ \\
Relative intensity noise $R I N$ & $-130 \mathrm{~dB} / \mathrm{Hz}$ \\
PD load resistor $R_{L}$ & $50 \Omega$ \\
Absolute temperature $T_{a b s}$ & $300 \mathrm{k}$ \\
Transmission distance $L$ & $2 \mathrm{~km}$ \\
Electron charge & $1.602 \times 10^{19}$ \\
Noise figure $F_{e}$ & $2 \mathrm{~dB}$ \\
Electrical filter bandwidth $B_{e}$ & $2 \mathrm{GhHz}$ \\
Number of carrier $N_{s}$ & 256 \\
Optical modulation index $m_{n}$ & 0.01 \\
Detector responsivity $R_{D}$ & $0.8 \mathrm{~A} / \mathrm{W}$ \\
\hline
\end{tabular}



Figure 3 Channel correlation coefficient $\rho_{12}$ versus separation distance $d_{12}$ with variation of turbulence strength regimes

correlation length $\rho_{c}$ is an important parameter which is useful in the choice of antenna spacings or making a quick judgment on whether channel correlations exist among component antennas. The obtained result shows the when the two channels are overlapped, they must be perfectly correlated. In fact, two separated channels are independent of each other when the separation distance is larger than channel correlation length $\rho_{c}$, and channel correlation is affected by turbulence strength. In addition, we consider the aperture averaging (AA) based on the previous literatures [14-16], the point receiver is defined as 
$D=\sqrt{L \lambda}$, $[4,11]$. Thus, the point receiver aperture size $D$ is approximate to $2 \mathrm{~cm}$ in this paper. The effect of AA on the system performance will be illustrated in Figure 6.

In Figure 4, we compare the system transmission performance of EGC and MRC combining scheme for OFDM-FSO link with different channel correlation coefficient, $\rho_{12}=(0.9,0.1)$. It is observed that performance of EGC receiver is very close to MRC receiver when system under same channel correlation coefficient. The results also shown that there is a $2 \mathrm{~dB}$ difference at average about BER $=10^{-6}$ for system with $\rho_{12}=0.1$ and $\rho_{12}=0.9$, respectively.

In this case, receiver aperture size is such as aperture averaging, $D=4 \mathrm{~cm}$. In uncorrelated channel $\left(\rho_{12}=0\right)$, each aperture deployment far apart, and separation distance beyond to the channel correlation length $\rho_{c}$, the transmission performance can be seen as an independent case. This point of view is also in contract we typically see in RF wireless communication $[13,14]$, where only the uncorrelated channel has a lower bit-error-ratio, channel fading can be reduced substantially. Our observations demonstrate

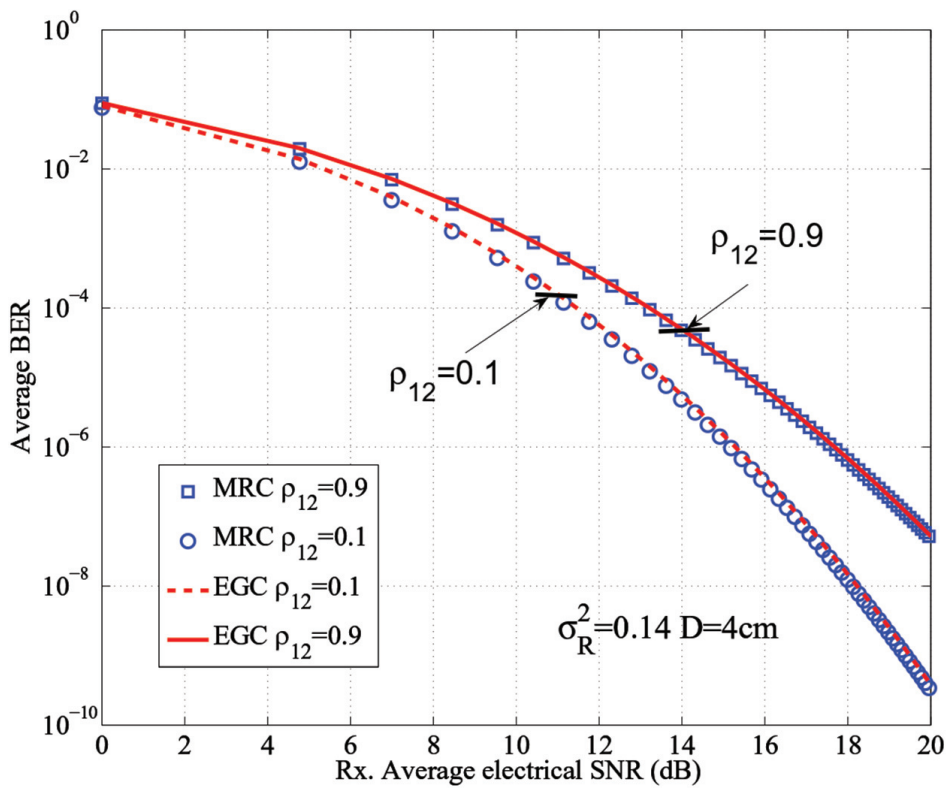

Figure 4 Average BER versus aver average electrical SNR of a OFDM-FSO link with dual diversity reception and MRC/EGC combining scheme over correlated Log-normal fading channel 
that efficient separation between the apertures is crucial to achieve the promised improvement of system from independent dual diversity reception (channel correlation coefficient $\rho_{12}$ approximate to zero, i.e. $\rho_{12}=0.1$ ).

In Figure 5, we plot the derived average BER versus the average electrical SNR for different types of modulation, i.e., BPSK, QPSK, 16-QAM, 64QAM, and adopt MRC combining scheme with channel correlation coefficient $\rho_{12}=0.1$ as a independent case and $\rho_{12}=0.9$ over weak turbulence channel, $\sigma_{R}^{2}=0.14$ with the point receiver aperture size $D=2 \mathrm{~cm}$. The performance of the proposed system is degraded when the correlation coefficient is high. However, for the average BER $=10^{-10}$, the BPSK with correlation co-efficient $\rho_{12}=0.1$ outperforms BPSK with correlation coefficient $\rho_{12}=0.9$ by approximately $5 \mathrm{~dB}$. These results indicate the importance of channel correlation and also demonstrate the effect of correlation on system performance. Moreover, the effect of modulation on the average BER in clearly apparent. When the average SNR $=14 \mathrm{~dB}$, for example, the average BER increases from $10^{-9}$ to $10^{-1.5}$ for QPSK and 64-QAM with same correlation coefficient $\rho_{12}=0.1$.

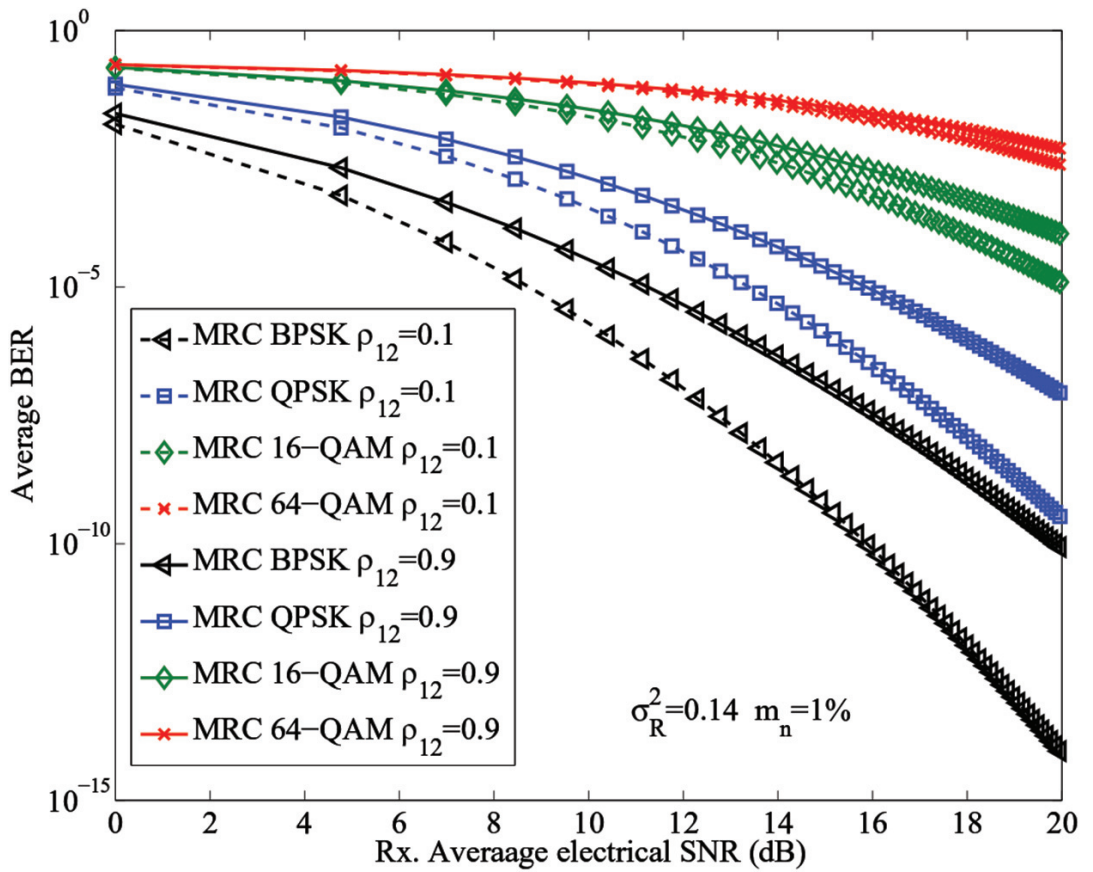

Figure 5 Average BER versus average electrical SNR of a OFDM-FSO link with dual diversity reception over correlated Log-normal fading channels 
In fact, the average BER performance of dual diversity reception OFDMFSO system with larger constellation size requires higher received power to accurately discriminate among the transmitted symbols; they are also sensitive to the turbulence due to the required precision in accurate constellation scaling at receiver.

To highlight the performance enhancement thanks to use of diversity receivers, we have shown in Figure 6 the system performance with ternary, dual and single reception in weak turbulence regime $\sigma_{R}^{2}=0.14$, and using MRC scheme for two different channel correlation coefficient $\rho_{12}=(0,0.9)$. In this analysis, the effect of aperture averaging on system performance have been considered, i.e. $D=4 \mathrm{~cm}$ for single reception. It should be noted that the collected area of single receiver aperture is equal to the sum of the diversity apertures area in this case. Before presenting the numerical results, we defined the correlation Log-normal distribution function (22) for the case of ternary reception model. Based on the equation (13), (23) and (25), the electrical SNR at the output of diversity combiner as $\gamma_{\text {total.MRC }}\left(I_{1}, I_{2}, I_{3}, X_{1}^{2}, X_{2}^{2}, X_{3}^{2}\right)=$ $\sum_{j=1}^{J} \gamma_{j}\left(X_{j}^{2}\right),(j=3)$, and average BER function as $\left\langle B E R_{\text {total }}\right\rangle=\int_{0}^{\infty} \int_{0}^{\infty}$ $\int_{0}^{\infty} f\left(X_{1}, X_{2}, X_{3}\right) B E R_{\text {total }}\left(X_{1}^{2}, X_{2}^{2}, X_{3}^{2}\right) d X_{1} d X_{2} d X_{3}$. The result clear

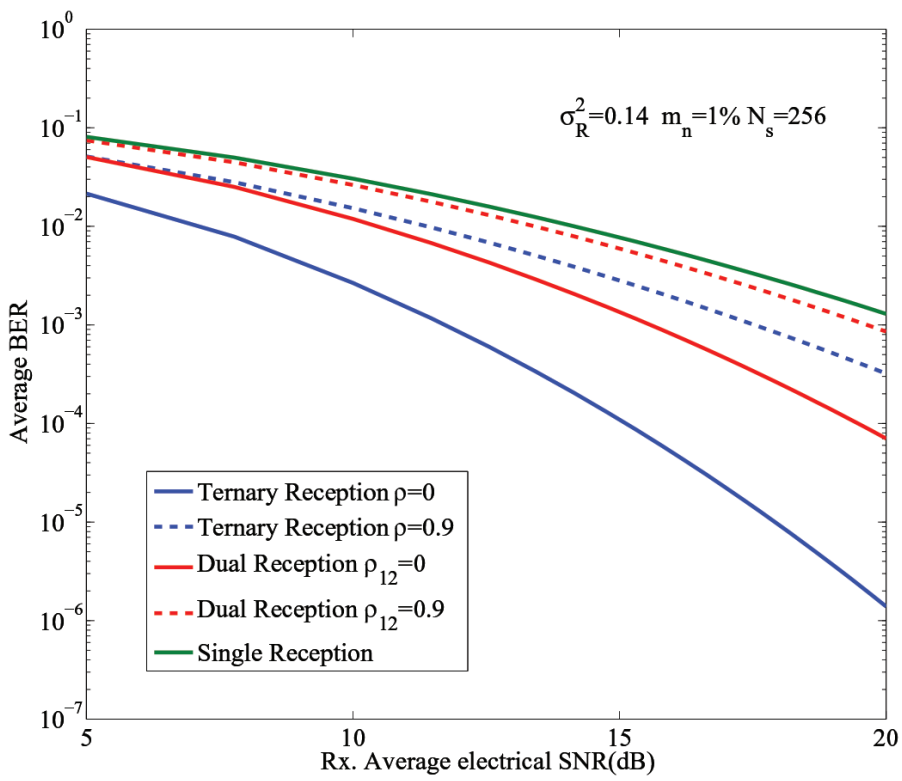

Figure 6 Performance comparison of ternary reception, dual diversity reception and single reception for OFDM-FSO link 
show that ternary reception system performance outperforms dual and single reception case. For the received electrical $\mathrm{SNR}=15 \mathrm{~dB}$, for example, the average BER increases from $10^{-4}$ for ternary reception, $10^{-3}$ for dual reception to $10^{-2}$ for single reception with AA under same channel correlation coefficient $\rho_{12}=0$. These result shown that selecting an diversity reception may increase the overall system performance and diversity technique can be obtained in practice through aperture averaging effect. Furthermore, we observe that there is a $5 \mathrm{~dB}$ difference at average BER $=10^{-4}$ for the ternary reception with channel correlation parameter $\rho_{12}=0$ and $\rho_{12}=0.9$, respectively. As pointed out above, the result indicate that increasing the channel correlation leads to system performance degradation.

The system's outage probability $P_{\text {out }}$ is analyzed using Equation(28). Figure 7 shows $P_{\text {out }}$ in terms of the threshold $S N R_{t h}$ for single and dual reception with QPSK in the weak turbulence regime with the scintillation index $\sigma_{R}^{2}=0.14$ and optical modulation index $m_{n}=0.01$. Two cases of channel correlation coefficient have been considered, $\rho_{12}=(0.1,0.9)$. It is observed that the use of a dual reception leads to better $P_{\text {out }}$, which outlines its conspicuous

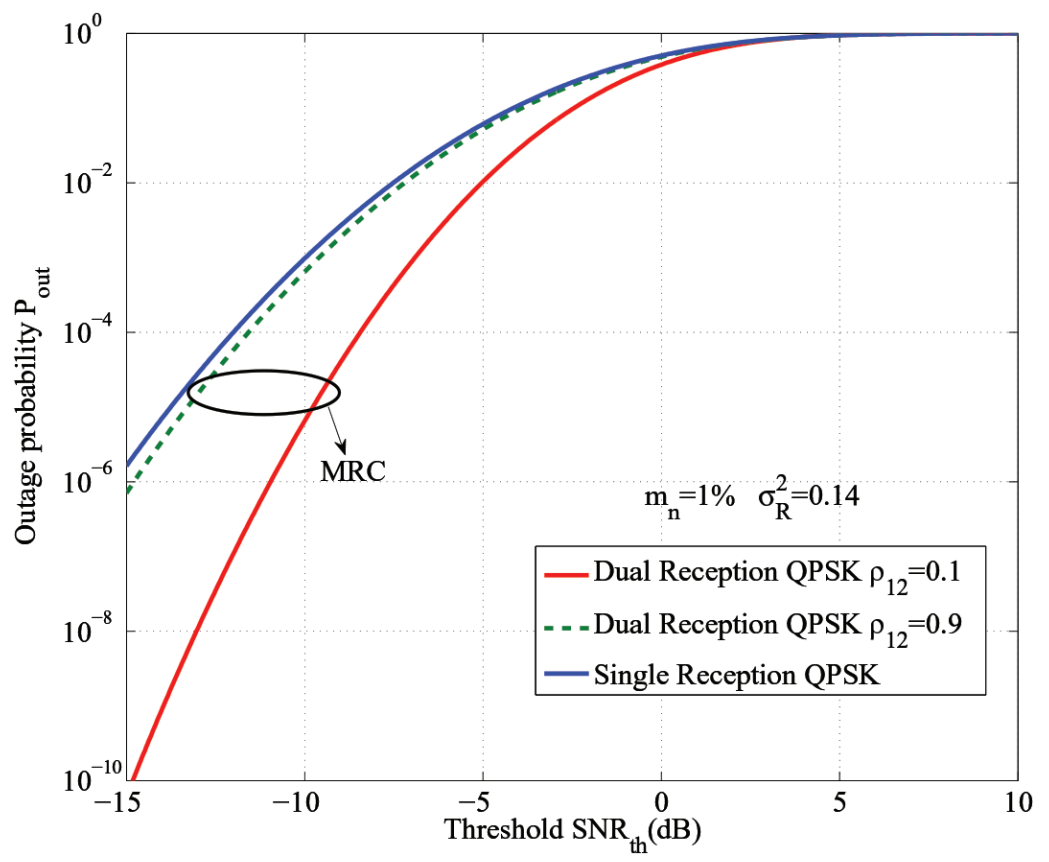

Figure 7 Performance of dual diversity reception with MRC and EGC for outage probability 
contribution to mitigating the turbulence-induced fading overcoming the performance degradation. For instance, for a $S N R_{t h}=-15 \mathrm{~dB}, P_{\text {out }}$ decreases from about $10^{-10}$ to $10^{-6}$ for a dual reception with $\rho_{12}=0.1$ and single reception, respectively. Now, we consider the case of the channel correlation, $\rho_{12}=(0.1,0.9)$ at the same outage probability $P_{\text {out }}=10^{-6}$, we obtained a $4 \mathrm{~dB}$ different for threshold $S N R_{t h}$ between $\rho_{12}=0.1$ and $\rho_{12}=0.9$ with a dual reception, respectively. The proposed system performance with channel correlation increasing leads to the performance degradation as the case of two channels are overlapped.

\section{Conclusion}

In this paper, we give a theoretical study of the channel correlation in the diversity reception OFDM-FSO system. In our analysis, we propose a novel transceiver architecture for atmospheric OFDM-FSO system with diversity reception over correlated fading channel. Based on the plane-wave model, we analyze and evaluate the channel correlation effects on the transmission performance of system over correlated Log-normal turbulence channel in terms of SNR, BER, outage probability and diversity combining scheme. Our numerical results demonstrate that diversity reception OFDM-FSO system performance is sensitive to the channel correlation, modulation format, aperture size and turbulence strength. The analysis results also represent spatial diversity as a helpful method to mitigate the channel fading and reduce the bit error of transmission. Furthermore, the evaluation of the proposed system behaviors outlines that the use of the diversity reception and OFDM technique can lead to substantial performance improvement, especially as high demand on transmission capacity becomes more important in next generation access networks. In the same time, our presented work can serve as preliminary guidelines to standard transceiver design for FSO communication system transmitting RF signals in the practical issue.

\section{References}

[1] H. Al-Raweshidy and S.Komaki, Eds. in Radio Over Fiber Technologies for Mobile Communications Networks, 1st ed. Artech House Publishers, 2002.

[2] Co-location longitudinally compatible interfaces for free space optical systems, ITU-T Rec. G.640, 2006. 
[3] A.Bekkali. Transmission Analysis of OFDM-Based Wireless Services Over Turbulent Radio-on-FSO Links Modeled by GammaCGamma Distribution. IEEE photonics journal, 2(3):510-520, 2010.

[4] J.Armstrong. OFDM for optical communications. J.Lightwave Technol., 27(3):189-204, 2009.

[5] L. C. Andrews and R. L. Phillips. in Laser Beam Propagation Through Random Media, Wellingham, WA: SPIE, 2005.

[6] Z.X. Chen. Channel correlation in aperture receiver diversity systems for free-space optical communication Journal of Optics, 2010.

[7] A.Bekkali, P.T.Dat, K.Kazaura, K. Wakamori, M.Matsumoto, T. Higashino, K. T-sukamoto and S.Komaki. Performance Evaluation of an Advanced DWDM RoFSO System for Transmitting Multiple RF Signals. IEICE TRANS. FUNDAMENTALS, 2009.

[8] S.Mohammad Navidpour. BER Performance of Free-Space Optical Transmission with Spatial Diversity IEEE Transactions on wireless communications, 26(8), 2007.

[9] X.Zhu and J. M. Kahn. Free-space optical communication through atmospheric turbulence channels. IEEE Trans. Commun., 50(8):1293-1300, 2002.

[10] D.Skraparlis. On the Effect of Correlation on the Performance of Dual Diversity Receivers in Lognormal Fading IEEE Communications Letters, 14(11), 2010.

[11] M. A. Khalighi. Fading Reduction by Aperture Averaging and Spatial Diversity in Optical Wireless Systems. J. OPT. COMMUN. NETW, 1(2), 2009.

[12] Z.X.Chen, S. Yu, T.Y. Wang, G.H. Wu and S.L.Wang Channel Correlation in Aperture Receiver Diversity Systems for Free-Space Optical Communication Journal of Optics, 14(2012), 125710(7pp), 2012.

[13] F.Bai, Y. W.Su, T.Sato Performance evaluation of a dual diversity reception base on OFDM RoFSO systems over correlated log-normal fading channel ITU Kaleidoscope Academic Conference, 263-268, 2014.

[14] S.Bloom. in The Physics of Free-Space Optics, AirFiber Inc., 2002.

[15] L. C. Andrews Aperture-averaging factor for optical scintillations of plane and spherical waves in the atmosphere JOSA A, 14(2012), 9(4), 597-600, 1992.

[16] J. A. Anguita, M. A. Neifeld and B. V. Vasic Spatial correlation and irradiance statistics in a multiple-beam terrestrial free-space optical communication link Applied Optics, 46(26), 6561-6571, 2007. 
[17] K. Kiasaleh Scintillation index of a multiwavelength beam in turbulent atmosphere JOSA A, 21(8), 1452-1454, 2004.

\section{Biographies}



Fan Bai received his B.E. degree in communication engineering from Changchun University of Science and Technology, Jilin Province, China, in 2007. From 2007 to 2010, he was with Beijing research and development center, ZTE Corporation, China, as system engineer. He received his M.Sc. degree in computer system and network engineering from the Waseda University, Japan, in 2013. He is currently pursuing his Ph.D. degree in Graduate School of Global Information and Telecommunication Studies (GITS), Waseda University, Japan. His research interests include optical wireless communications, optical fiber communications and digital signal processing. He is a student member of IEICE.

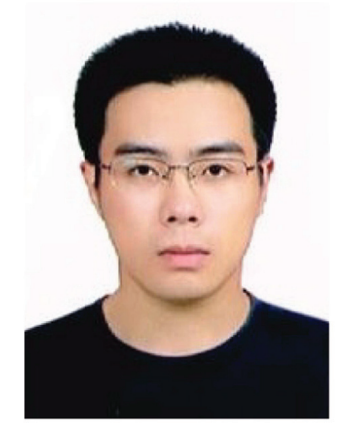

Yuwei Su was born in 1989 and received the B.E. degree from University of Electronic Science and Technoloy of China, Chengdu, China, in 2012. 
He received the M.S. Degree from Waseda University, Japan. He is currently working toward the Ph.D. degree in the School of Fundamental Science and Engineering, Waseda University. His areas of interests include wireless communication, free space optics and optical science and technology.

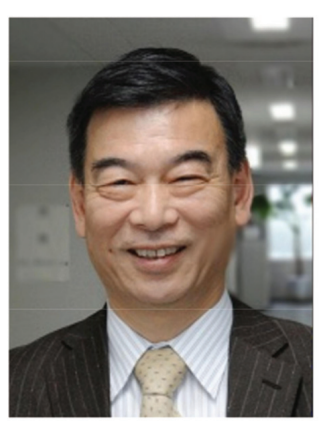

Takuro Sato, professor. He received the Ph.D dgree in electronics from Niigata University, Japan, in 1994. From 1973 to 1995, he was with research and development laboratories, OKI electric Co., Ltd, Japan, as project leader. From 1995 to 2004, he was with department of information and electronics engineering, Niigata institute of technology, Japan, as professor. From 2004 to now, he was with graduate school of globe information and telecommunication studies as professor, Waseda university, Japan. His research interests include wireless communication, ICN/CCN, smart grid. Prof. Sato is a IEEE fellow, IEICE fellow. 
